\title{
Effect of intervention in the diacylglycerol-protein kinase $C$ signaling pathway on JNK1 expression and its downstream signaling in diabetic cardiomyopathy
}

\author{
XIAOLIANG LIU ${ }^{1}$, FANG QI $^{2}$ and WEI WU ${ }^{1}$ \\ ${ }^{1}$ Department of Emergency Medicine, The First Affiliated Hospital of China Medical University; \\ ${ }^{2}$ Department of General Surgery, Fengtian Hospital Affiliated to Shenyang Medical College, \\ Shenyang, Liaoning 110000, P.R. China
}

Received July 7, 2013; Accepted December 10, 2013

DOI: $10.3892 / \mathrm{mmr} .2014 .1904$

\begin{abstract}
This study aimed to investigate the expression of signaling molecules, such as c-Jun N-terminal kinase 1 (JNK1) and insulin receptor substrate 1 (IRS1), in the myocardium of diabetic rats following intervention in the diacylglycerol-protein kinase C (DAG-PKC) signal transduction pathway. The rats were divided into three groups, the diabetic model, control and breviscapine-treated diabetes (intervention) group. Following modeling and drug treatment, hematoxylin and eosin (HE) and Masson staining and electron microscopy were used to observe the pathological changes in the rat myocardium. The expression of PKC- $\beta 2$, JNK1, and IRS1 was assessed in rat myocardium by immunohistochemistry and quantitative polymerase chain reaction (qPCR). The expression levels of PKC- $\beta 2$, JNK1, phosphorylated JNK (p-JNK) and IRS1 in the diabetic model group were significantly higher than those in the control group. Furthermore, compared with the diabetic model group, expression levels of PKC- $\beta 2$, JNK1, p-JNK and IRS1 were significantly reduced following intervention in the DAG-PKC signal transduction pathway. The DAG-PKC pathway may affect downstream signaling through JNK1 (the common signal point of the G-protein receptor pathway and insulin receptor pathway at the cell membrane) to result in the occurrence and development of diabetic cardiomyopathy (DCM). The series of signal points DAG-PKC-JNK1-IRS1-Akt/PKB-mTOR-p70S6K1 is a potential pathway for inducing DCM by DAG-PKC signal transduction. Enhanced expression of JNK1, p-JNK and IRS1 may accelerate diabetic myocardial fibrosis.
\end{abstract}

Correspondence to: Professor Wei Wu, Department of Emergency Medicine, The First Affiliated Hospital of China Medical University, Nanjing north Street No. 155, Shenyang, Liaoning 110000, P.R. China

E-mail: xlwwcn@yeah.net

Key words: DAG-PKC signal transduction pathway, diabetic cardiomyopathy, JNK1

\section{Introduction}

Patients with diabetes mellitus (DM) present with various pathological conditions, including accelerating atherosclerosis, cardiac autonomic neuropathy, myocardial protein changes (1), myocardial oxidation (2) and intrinsic cardiomyopathy $(3,4)$. These cardiac diseases associated with DM $(5)$ are known as diabetic cardiomyopathy (DCM). Previous studies on the pathogenesis of DCM mainly focused on factors such as myocardial metabolism, calcium transit and abnormal free oxygen radicals. The mechanisms of intracellular signal transduction and cell function regulation in DCM are of increasing interest and are the subject of current studies. The excessive activation of the diacylglycerol-protein kinase C (DAG-PKC) pathway has a pivotal role in intracellular signal transduction systems. The activation of the DAG-PKC pathway has been shown to be an important mechanism in the early pathological changes during the formation of diabetic myocardial cells. This study, based on previous investigations, used streptozotocin (STZ)-induced diabetic rats as a model to investigate the myocardial pathological changes of early diabetes, and the correlation between PKC- $\beta 2$ and c-Jun N-terminal kinase 1 (JNK1) was analyzed. The role of the enhancement of signaling agents, such as JNK1 and the insulin receptor substrate 1 (IRS1), in the occurrence and development of DCM was observed. The intervention in the expression of signaling agents, such as JNK1 and IRS1, provided a theoretical basis for signal transduction inhibition for the prevention and treatment of DCM.

\section{Materials and methods}

Modeling of diabetes and grouping. A total of 60 specific pathogen-free Sprague Dawley rats provided by the Experimental Animals Ministry of China Medical University (Shenyang, Liaoning, China) were randomly divided into control, diabetic model and breviscapine-treated diabetes (20 $\mu \mathrm{g} / \mathrm{kg} /$ day, intraperitoneal injection) groups, with 20 rats in each group. Following normal feeding and subsequent fasting for $24 \mathrm{~h}$ with free access to water, the rats of the experimental group were treated with $60 \mathrm{mg} / \mathrm{kg} \mathrm{STZ} \mathrm{(Sigma,} \mathrm{St.} \mathrm{Louis,}$ 
MO, USA) dissolved in $0.1 \mathrm{~mol} / 1$ citrate buffer ( $\mathrm{pH} 4.5$ ) by left intraperitoneal injection with moderate action to avoid damaging internal organs and blood vessels. The rats in the control group were injected with an equivalent volume of citrate buffer. Urine and blood glucose levels were assessed after $72 \mathrm{~h}$. The rats with blood glucose $>11.1 \mathrm{mmol} / 1$ with fasting, blood glucose $>16.7 \mathrm{mmol} / \mathrm{l}$ without fasting, qualitative urine glucose $\geq+++$, polydipsia polyphagia and increasing urine output were defined as the DM model. The rats in the intervention group were treated with breviscapine $(20 \mu \mathrm{g} / \mathrm{kg} /$ day; Yunnan Natural Medicine Pharmaceutical Co., China) through intraperitoneal injection. The rats in the control and the model groups were injected with an equivalent volume of solvent. All rats were fed a standard diet and provided with freely available drinking water during the entire experiment, which had a duration of eight weeks. There was no insulin therapy throughout the entire experiment, and the rats were sacrificed at appropriate time-points. This study was performed in strict accordance with the recommendations from the Guide for the Care and Use of Laboratory Animals of the National Institutes of Health (NIH). The protocol for the animal use was reviewed and approved by the Institutional Animal Care and Use Committee (IACUC) of China Medical University.

Determination of blood glucose and body weight. Following fasting for $12 \mathrm{~h}$, the entire blood of the rat tail peripheral capillary was extracted to detect the fasting blood sugar using the Roche Accu-CHEK active blood glucose meter (Roche Diagnostics, GmbH, Mannheim, Germany). The body weight of the rats was measured using scales once per week.

Determination of the entire heart and left ventricular weights. Ten rats were randomly selected from the three groups during the eight-week treatment. All rats were anesthetized with $2 \mathrm{ml} \mathrm{3 \%}$ sodium pentobarbital by intraperitoneal injection following being weighed. The sternum was opened to expose the heart, which was separated from the aortic roots, dried on filter paper following rinsing with cold saline, and then weighed. The left ventricle was then separated and weighed. The weight ratio of heart and body $(\mathrm{H} / \mathrm{W}$; entire heart weight/body weight) and the left ventricular mass index (LVMI; left ventricular weight/body weight) were calculated, respectively.

Hematoxylin and eosin (HE) and Masson staining. The pathological specimens were fixed for $24 \mathrm{~h}$ with $10 \%$ neutral formalin by the conventional method, dehydrated with ethanol gradients, embedded in paraffin and stained with HE. Positive areas of the stained collagen were quantified using Image-Pro ${ }^{\circledR}$ Plus 6.0 medical image analysis software (Media Cybernetics, Inc., Rockville, MD, USA) and were blindly selected, and five fields of view were selected for each slice. The myocardial collagen volume fractions (CVFs) were calculated using the following formula: CVF $(\%)=$ collagen area/ full field area x100, and the averages were calculated.

Observation by electron microscopy. A total of $1 \mathrm{~mm}^{3}$ myocardial tissue was fixed in $2.5 \%$ glutaraldehyde, rinsed with phosphate-buffered saline (PBS), fixed with $1 \%$ osmium tetroxide, dehydrated with ethanol by the conventional method and embedded in epoxy resin. Tissue was then sectioned (70 $\mathrm{nm}$ ), dyed with uranyl acetate and lead citrate, and observed and filmed using JME-1200EX electron microscopy (Japan Electron Optics laboratory Co., Ltd., Tokyo, Japan).

Immunohistochemistry. Sections of rat left ventricles $(0.5 \times 0.5 \mathrm{~cm})$ were fixed in $4 \%$ paraformaldehyde for $24 \mathrm{~h}$, embedded in conventional paraffin, and sectioned into $4 \mathrm{pm}$ slices. PKC- $\beta 2$, JNK1 and IRS-1 were immunohistochemically quantified. The expression levels of JNK1, IRS-1 and PKC- $\beta 2$ were detected using the StreptAvidin-Biotin Complex (SABC) method. The morphological image was analyzed using systems software (Xiamen City Bao Technology Co., Ltd., Xiamen, China), five fields of view were randomly tested for each slice, and then the average optical density value (MOD) was calculated for each sample. The MOD values were used as semi-quantitative parameters for the expression levels of JNK1, IRS-1 and PKC- $\beta 2$.

Quantitative polymerase chain reaction ( $q P C R)$. Myocardial left ventricular free wall $(100 \mathrm{mg})$ was placed in diethylpyrocarbonate (DEPC) water prepared in advance, prior to being numbered and frozen in liquid nitrogen, and stored at $-80^{\circ} \mathrm{C}$ to measure the expression of JNK1 mRNA and IRS-1 mRNA in the left ventricle. Total RNA was extracted using a one-step method according to the instructions for the TRIzol ${ }^{\circledR}$ reagent (Invitrogen Life Technologies, Carlsbad, CA, USA). In order to determine RNA concentration and purity, $1 \mu 1 \mathrm{RNA}$ sample was obtained and $79 \mu \mathrm{l}$ DEPC water was added to detect OD260 and OD280 values using an ultraviolet spectrophotometer. A ratio between the two of 1.8 to 2.0 suggested that the RNA purity of the sample was sufficient. cDNA was then synthesized using reverse transcription, in accordance with the kits' instructions (cDNA synthesis kits; Beijing Genomics Corporation, Beijing, China). The qPCR reaction was performed using an ABI 7500 thermal cycler (Biometra, Göttingen, Germany). cDNA was then synthesized using reverse transcription with mRNA.

Statistical analysis. The experimental data were processed using SPSS 13.0 statistical software (SPSS, Inc., Chicago, IL, USA). The test results are expressed as the mean \pm standard deviation, and analyzed using analysis of variance or the Student's t-test. $\mathrm{P}<0.05$ indicated a significant difference. $\mathrm{P}<0.01$ indicated a highly significant difference.

\section{Results}

General indexes. Blood glucose levels were significantly increased in the diabetic model group and the breviscapine-treated diabetes group compared with the control group $(\mathrm{P}<0.01)$ and the body weight was significantly reduced $(\mathrm{P}<0.01)$, while blood glucose levels were not significantly different between the diabetic model group and the breviscapine-treated diabetes group, and the body weight in the breviscapine-treated diabetes group was higher than that of the diabetic model group $(\mathrm{P}<0.05)$. The left ventricular weight, the ratio of heart and body weight and the left ventricular mass of the diabetic model and breviscapine-treated diabetes group were significantly higher than those of the control group (all 
Table I. Index comparison of rat blood glucose, body weight, entire heart weight, left ventricular weight, ratio of heart and body weight and left ventricular mass among the treatment groups.

\begin{tabular}{lccccccc}
\hline Group & $\begin{array}{c}\text { Cases } \\
(\mathrm{n})\end{array}$ & $\begin{array}{c}\text { Fasting } \\
\text { blood-glucose } \\
(\mathrm{mmol} / \mathrm{l})\end{array}$ & $\begin{array}{c}\text { Body } \\
\text { weight }(\mathrm{g})\end{array}$ & $\begin{array}{c}\text { Entire } \\
\text { heart } \\
\text { weight }(\mathrm{g})\end{array}$ & $\begin{array}{c}\text { Left } \\
\text { ventricular } \\
\text { weight }(\mathrm{g})\end{array}$ & $\begin{array}{c}\text { Ratio of heart } \\
\text { and body } \\
\text { weight }\left(10^{-3}\right)\end{array}$ & $\begin{array}{c}\text { Left } \\
\text { ventricular } \\
\text { mass }\left(10^{-3}\right)\end{array}$ \\
\hline Control & 10 & $7.00 \pm 1.1$ & $312.5 \pm 17.08$ & $0.84 \pm 0.17$ & $0.74 \pm 0.04$ & $2.7 \pm 0.06$ & $2.37 \pm 0.02$ \\
Diabetic & 10 & $26.8 \pm 2.9^{\mathrm{a}}$ & $203.8 \pm 4.79^{\mathrm{a}, \mathrm{b}}$ & $0.79 \pm 0.39^{\mathrm{a}, \mathrm{b}}$ & $0.59 \pm 0.02^{\mathrm{a}, \mathrm{b}}$ & $3.6 \pm 0.17^{\mathrm{a}, \mathrm{b}}$ & $2.94 \pm 0.07^{\mathrm{a}, \mathrm{b}}$ \\
Intervention & 10 & $27.9 \pm 1.2$ & $247.5 \pm 18.9$ & $0.73 \pm 0.18$ & $0.61 \pm 0.05$ & $3.2 \pm 0.13$ & $2.49 \pm 0.01$ \\
\hline
\end{tabular}

${ }^{\mathrm{a}} \mathrm{P}<0.01$ compared with control group; ${ }^{\mathrm{b}} \mathrm{P}<0.01$ compared with the intervention group.

Table II. Comparison of rat myocardial collagen volume fraction and perivascular collagen areas among the treatment groups.

\begin{tabular}{lccc}
\hline Group & Cases $(\mathrm{n})$ & CVF $(\%)$ & PCVA (\%) \\
\hline Control & 10 & $3.19 \pm 0.57$ & $7.15 \pm 1.06$ \\
Diabetic & 10 & $9.57 \pm 0.94^{\mathrm{a}, \mathrm{b}}$ & $18.32 \pm 4.41^{\mathrm{a}, \mathrm{b}}$ \\
Intervention & 10 & $6.41 \pm 1.58$ & $12.14 \pm 0.22$ \\
\hline
\end{tabular}

${ }^{a} \mathrm{P}<0.01$ compared with control group; ${ }^{\mathrm{b}} \mathrm{P}<0.01$ compared with the intervention group. CVF, collagen volume fraction; PCVA, perivascular collagen areas.

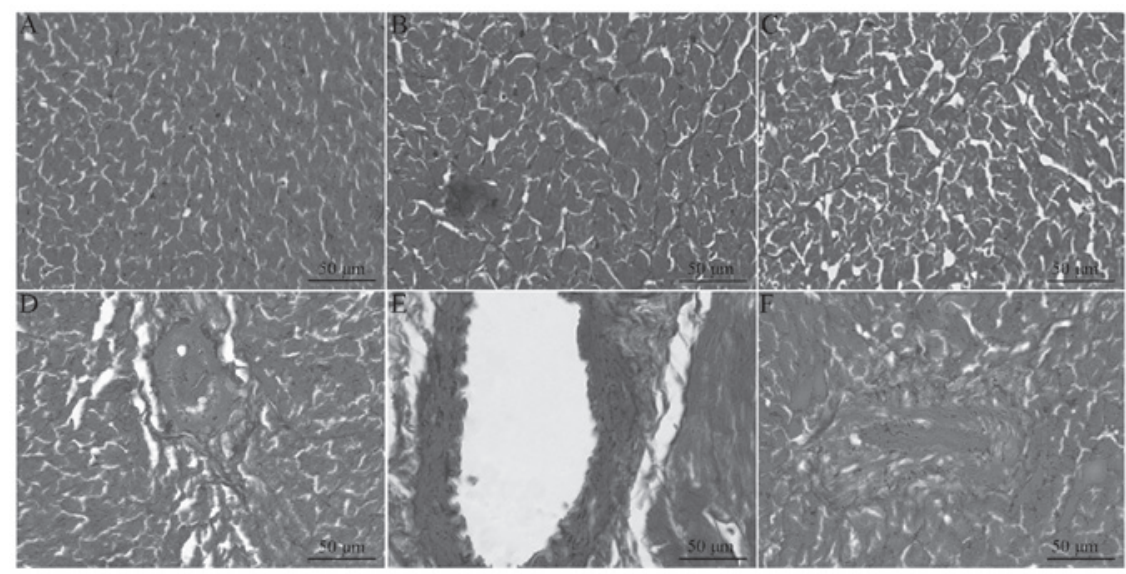

Figure 1. Masson staining of rat myocardial tissue and the small blood vessels around the heart in each group (magnification, $\mathrm{x} 400$ ). (A-C) Masson staining of myocardial tissue in the (A) normal, (B) diabetic and (C) intervention groups. (D-F) Masson staining of small blood vessels around the heart in the (D) normal,

(E) diabetic and (F) intervention groups.

$\mathrm{P}<0.01$ ), and these differences between the diabetic model and breviscapine-treated diabetes groups were also significant (all $\mathrm{P}<0.01$ ) (Table I).

Results of myocardium interstitial fibrosis. According to the HE staining, the myocardium was clearly striated and regularly arrayed in the normal rats, while the diabetic model group featured disordered cell arrays and focal necrosis, which was improved following treatment with breviscapine. The collagenous fibers were blue-green in the Masson staining, and the muscle fibers were red. The collagen was evenly distributed in the normal rats. In the diabetes group, the myocardial collagenous fibers were significantly increased, disordered, unevenly distributed and closely arranged around the myocardial cells, and were observed to surround the small vessels. The myocardial collagenous fibers were significantly decreased in the breviscapine-treated diabetes group. The myocardial CVF and perivascular collagen areas (PVCA) were significantly increased, with the increase in PVCA being more marked, 2.5-fold that of the normal amount, and the CVF was about two-fold that of the normal amount (Table II; Fig. 1).

Observation by electron microscopy. The rat myocardial cells in the normal group exhibited numerous regularly arranged myofilaments with clear bright-dark zones. The mitochondria were normal, round or oval, contained obviously advanced ridges and were concentrated within the cell. 


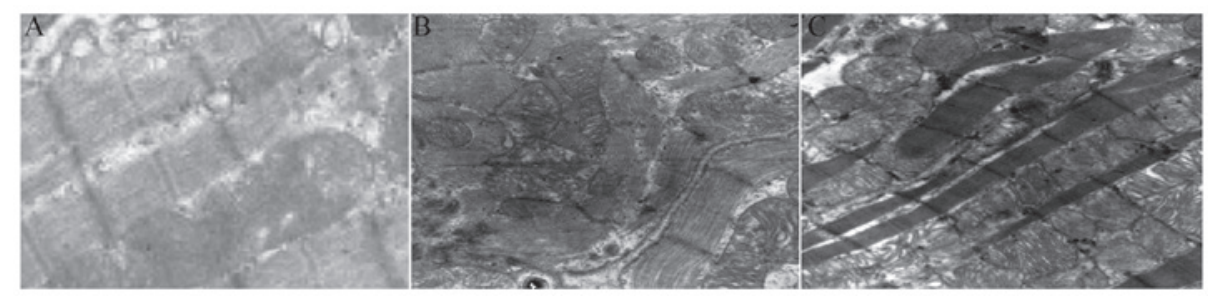

Figure 2. Transmission electron microscopy images of rat myocardial tissue in each group (magnification, x5,000). (A) Normal, (B) diabetic and (C) intervention groups.

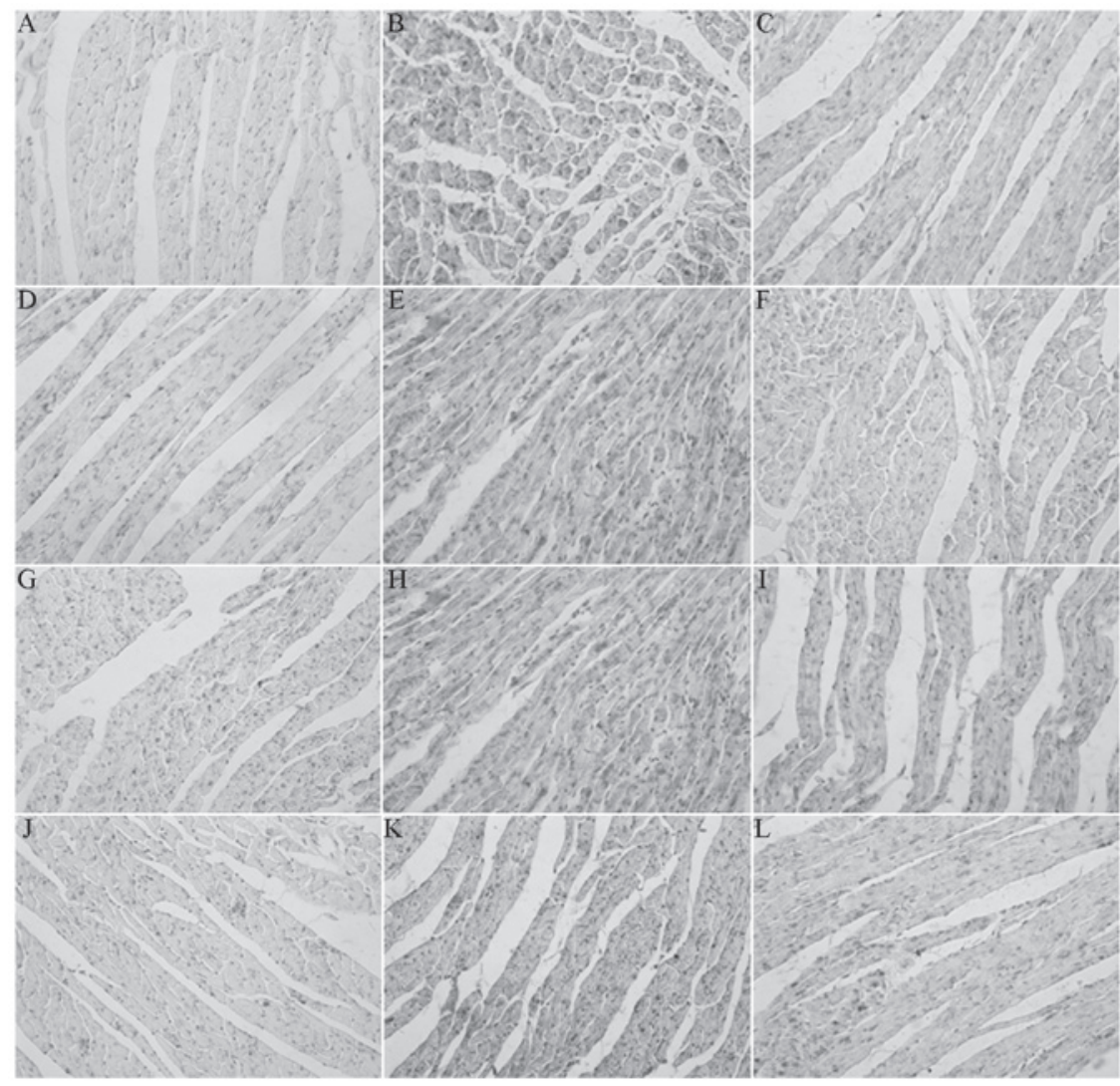

Figure 3. Expression of PKCII, JNK1, p-JNK1 and IRS1 proteins in rat myocardial tissues in each group (immunohistochemistry; magnification, x200). (A-C) Positive expression of PKCII protein for myocardial tissue in the (A) normal, (B) diabetic and (C) intervention groups. (D-F) Positive expression of JNK1 protein for myocardial tissue in the (D) normal, (E) diabetic and (F) intervention groups. (G-I) Positive expression of p-JNK1 protein for myocardial tissue in the (G) normal, (H) diabetic and (I) intervention groups. (J-L) Positive expression of IRS1 protein for myocardial tissue in the (J) normal, (K) diabetic and (L) intervention groups. PKCII, protein kinase C II; JNK1, c-Jun N-terminal kinase 1; p-JNK1, phosphorylated JNK1; IRS1, insulin receptor substrate 1.

Regularly arranged intercalated disc connections were found in the myocardial cells. The muscle fibrils of the myocardial cells in the diabetes model group were significantly reduced, the myofilaments were arranged in a disordered and sparse manner, and certain myofilaments were fragmented, tortuous, locally dissolved and contained missing and unclear bright-dark zones. The mitochondria were arranged in a disordered manner, and certain mitochondria were obviously swollen, and contained ridges that were widened, fragmented or even missing. In addition, the density of the stroma in these mitochondria was reduced and vacuoles had formed in the stroma. Following the intervention, the number of the muscle fibrils was increased compared with the diabetes model group, the myofilaments were arranged regularly, the mitochondria were slightly swollen, the intercristal spaces were slightly widened, and amalgamated and fractured vacuole structures were reduced (Fig. 2).

Immunohistochemistry. The images of three groups were captured using the micro-imaging analytical system (MetaMorph/DP10/BX41) to obtain the optical density values. The MODs of PKC- $\beta 2$, JNK1, p-JNK and IRS1 in the diabetes model groups were significantly higher than those in the control group, and the expression levels in the breviscapine-treated diabetes group were reduced compared with the diabetes model group, suggesting statistical significance $(\mathrm{P}<0.05)$ (Table III, Fig. 3).

$q P C R$. The levels of JNK1 and IRS-1 mRNA expressed in the myocardial cells of the normal group were low, while 
Table III. Comparison of the average optical density value for PKC, JNK1, p-JNK and IRS-1 in rat myocardium among the treatment groups.

\begin{tabular}{lcllll}
\hline Group & Cases $(\mathrm{n})$ & PKC & JNK1 & p-JNK & IRS1 \\
\hline Control & 10 & $0.283 \pm 0.051$ & $0.132 \pm 0.015$ & $0.149 \pm 0.005$ & $0.193 \pm 0.033$ \\
Diabetic & 10 & $0.408 \pm 0.013^{\mathrm{a}, \mathrm{b}}$ & $0.387 \pm 0.064^{\mathrm{a}, \mathrm{b}}$ & $0.392 \pm 0.030^{\mathrm{a}, \mathrm{b}}$ & $0.380 \pm 0.071^{\mathrm{a}, \mathrm{b}}$ \\
Intervention & 10 & $0.352 \pm 0.009$ & $0.243 \pm 0.111$ & $0.201 \pm 0.021$ & $0.238 \pm 0.014$ \\
\hline
\end{tabular}

Results are presented as the mean \pm standard deviation. ${ }^{\mathrm{a}} \mathrm{P}<0.01$ compared with control group; ${ }^{\mathrm{b}} \mathrm{P}<0.01$ compared with the intervention group. PKC, protein kinase C; JNK1, c-Jun N-terminal kinase 1; p-JNK, phosphorylated JNK; IRS1, insulin receptor substrate 1.
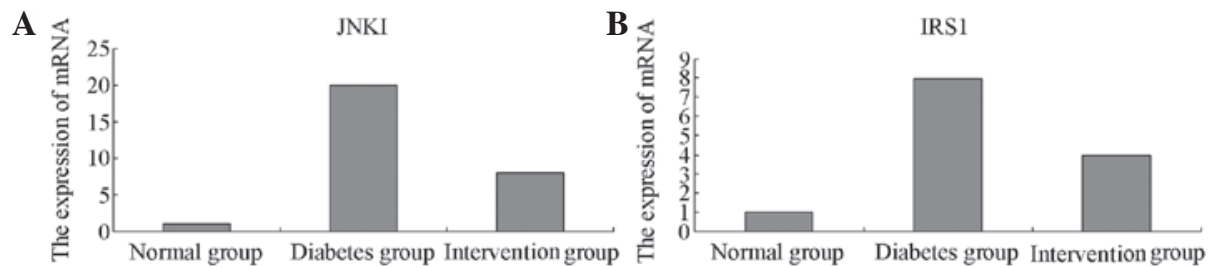

Figure 4. mRNA expression levels of JNK1 and IRS1 in rat myocardium in each group. (A) mRNA expression of JNK1. (B) mRNA expression of IRS1. "P $<0.01$ compared with control group; "P<0.05 compared with the intervention group. JNK1, c-Jun N-terminal kinase 1; IRS1, insulin receptor substrate 1.

significantly increasing in the diabetes model group (30-fold or 20 -fold the level of the control group) $(\mathrm{P}<0.01)$. Following treatment with breviscapine, the expression levels of the JNK1 mRNA and IRS-1 mRNA were markedly downregulated (Fig. 4).

\section{Discussion}

Previous studies on the pathogenesis of DCM mainly focused on myocardial cell metabolism, calcium ion transport and oxygen radical abnormalities $(6,7)$. The study of intracellular signal transduction and cell function regulation in DCM has drawn increasing focus, and excessive activation of the DAG-PKC pathway is suggested to have a pivotal role in the pathogenesis. The activation of PKC was shown to be an important mechanism in the early cardiac dysfunction caused by diabetes (8). PKC regulates the cascaded downstream signals in the DAG-PKC pathway by the diacylglycerol kinase subtype DGK $\zeta$ controlling the DAG levels. The cardiomyocytes of mice with Type I DM induced by STZ were analyzed (9), and a causal connection between the translocation from the endochylema to the cytomembrane of the PKC- $\beta$ and $-\delta$ subtypes and a reduction in the heart's blood-pumping function or an improvement in myocardium interstitial fibrosis were found. However, in DGK $\zeta$ transgenic DM mice, the translocation from the endochylema to the cytomembrane of the PKC- $\beta$ and $-\delta$ subtypes was significantly reduced, and there was no significant left ventricular systolic dysfunction or myocardial fibrosis (9). In the present study, the expression of PKC- $\beta 2$ in the myocardium of the diabetic group was significantly higher than that in the control and intervention groups. The preliminary applications of the highly selective PKC- $\beta 2$ inhibitor, LY333531, have further defined the role of PKC- $\beta 2$ in diabetic microangiopathy (10). The large number of PKC isozymes may have various roles in the remodeling of the myocardium, including the possibility of PKC- $\beta 2$ affecting the downstream signaling by activating JNK1 (11-14). Increasing the expression of downstream signaling agents, including PKC- $\beta 2$, JNK1, p-JNK and IRS1, has been shown to accelerate the progression of diabetic myocardial interstitial fibrosis (15).

This study revealed that, compared with the control group, the expression of JNK1, p-JNK1 and IRS1 in the diabetic group was significantly increased $(\mathrm{P}<0.01)$, while their expression was reduced following the inhibition of the DAG-PKC pathway. The activated JNK1 not only had a role in the mitogen-activated protein kinase (MAPK) signaling pathway, but also phosphorylated IRS1, which was stimulated by insulin or insulin-like growth factor (IGF-1). Accordingly, JNK1 may be able to regulate IRS1 signaling in the insulin/IGF1 pathway comprising phosphatidylinositol 3-kinase (PI3K) activation by IRS1, resulting in cardiac hypertrophy and heart failure (16) through further activation of the serine/threonine protein kinase/protein kinase B (Akt/PKB) pathway by PI3K. The latest study revealed a role for mammalian target of rapamycin (mTOR) through phosphorylation at mTORser ${ }^{2448}$ in the Akt/ PKB pathway; however, this complex pathway has yet to be fully elucidated (17). In this study, only one of the effects was revealed, which is that mTORser ${ }^{2448}$ phosphorylation activates ribosomal protein S6 kinase 1 (p70S6K1), promotes the generation of the hypoxia-inducible factor 1 (HIF1) and vascular endothelial growth factor (VEGF), and accelerates cardiac hypertrophy (18-20).

In conclusion, the DAG-PKC pathway may affect downstream signaling through JNK1 (the common signal point of the G-protein receptor pathway and the insulin receptor pathway at the cell membrane), which results in the occurrence and development of DCM. The series of signal points DAG-PKC-JNK1-IRS1-Akt/PKB-mTOR-p70S6K1 may be a potential pathway for inducing DCM via the DAG-PKC signal 
transduction pathway. Current challenges include controlling high-risk factors, such as the DAG-PKC signal transduction systems and significant increases in the expression of JNK1, p-JNK1 and IRS1, in DCM. If the general mechanism of the signal transduction during the formation and progression of DCM is elucidated and targeted interventions are performed, the incidence of DCM may be reduced and the patients' quality of life may be improved.

\section{Acknowledgements}

This study was supported by the Chinese Pharmaceutical Science Development Prize (No. L2012057) and science fund of the First Hospital of China Medical University (No. FSFH1207).

\section{References}

1. Dhalla NS, Rangi S, Zieroth $\mathrm{S}$ and $\mathrm{Xu}$ YJ: Alterations in sarcoplasmic reticulum and mitochondrial functions in diabetic cardiomyopathy. Exp Clin Cardiol 17: 115-120, 2012.

2. Hamblin M, Friedman DB, Hill S, Caprioli RM, Smith HM and Hill MF: Alterations in the diabetic myocardial proteome coupled with increased myocardial oxidative stress underlies diabetic cardiomyopathy. J Mol Cell Cardiol 42: 884-895, 2007.

3. Retnakaran R and Zinman B: Type 1 diabetes, hyperglycaemia, and the heart. Lancet 371: 1790-1799, 2008.

4. Chavali V, Tyagi SC and Mishra PK: Predictors and prevention of diabetic cardiomyopathy. Diabetes Metab Syndr Obes 6: $151-160,2013$

5. Otsui K, Inoue N, Tamagawa A and Onishi K: A case of mitochondrial cardiomyopathy with restrictive transmitral filling pattern. Int Med Case Rep J 5: 19-22, 2012.

6. Shi FH, Cheng YS, Dai DZ, Peng HJ, Cong XD and Dai Y: Depressed calcium-handling proteins due to endoplasmic reticulum stress and apoptosis in the diabetic heart are attenuated by argirein. Naunyn Schmiedebergs Arch Pharmacol 386: 521-531, 2013.

7. Yildirim SS, Akman D, Catalucci D and Turan B: Relationship between downregulation of miRNAs and increase of oxidative stress in the development of diabetic cardiac dysfunction: junctin as a target protein of miR-1. Cell Biochem Biophys: 13 May 2013 (Epub ahead of print). doi: 10.1007/s12013-013-9672-y.
8. Durgan DJ, Smith JK, Hotze MA, et al: Distinct transcriptional regulation of long-chain acyl-CoA synthetase isoforms and cytosolic thioesterase 1 in the rodent heart by fatty acids and insulin. Am J Physiol Heart Circ Physi 290: H2480-H2497, 2006.

9. Bilim O, Takeishi Y, Kitahara T, et al: Diacylglycerol kinase zeta inhibits myocardial atrophy and restores cardiac dysfunction in streptozotocin-induced diabetes mellitus. Cardiovasc Diabetol 7: 2, 2008.

10. Arikawa E, Ma RC, Isshiki K, et al: Effects of insulin replacements, inhibitors of angiotensin, and PKCbeta's actions to normalize cardiac gene expression and fuel metabolism in diabetic rats. Diabetes 56: 1410-1420, 2007.

11. Asghar O, Al-Sunni A, Khavandi K, et al: Diabetic cardiomyopathy. Clin Sci (Lond) 116: 741-760, 2009.

12. Liu X, Wang J, Takeda N, Binaglia L, Panagia V and Dhalla NS: Changes in cardiac protein kinase $\mathrm{C}$ activities and isozymes in streptozotocin-induced diabetes. Am J Physiol 277: E798-E804, 1999.

13. Ishii H, Koya D and King GL: Protein kinase $\mathrm{C}$ activation and its role in the development of vascular complications in diabetes mellitus. J Mol Med (Berl) 76: 21-31, 1998.

14. Mebazaa A, Gheorghiade M, Zannad FM and Parrillo JE (eds): Acute Heart Failure. Springer-Verlag, London, 2008.

15. Lei S, Li H, Xu J, et al: Hyperglycemia-induced protein kinase $C \beta 2$ activation induces diastolic cardiac dysfunction in diabetic rats by impairing caveolin-3 expression and Akt/eNOS signaling. Diabetes 62: 2318-2328, 2013.

16. Liang Q and Molkentin JD: Redefining the roles of p38 and JNK signaling in cardiac hypertrophy: dichotomy between cultured myocytes and animal models. J Mol Cell Cardiol 35: 1385-1394, 2003.

17. Gordon BS, Kazi AA, Coleman CS, Dennis MD, Chau V, Jefferson LS and Kimball SR: RhoA modulates signaling through the mechanistic target of rapamycin complex 1 (mTORC1) in mammalian cells. Cell Signal: Dec 2, 2013 (E-pub ahead of print).

18. Spangenburg EE: Changes in muscle mass with mechanical load: possible cellular mechanisms. Appl Physiol Nutr Metab 34: 328-335, 2009.

19. Vary TC, Deiter GG and Lantry R: Chronic alcohol feeding impairs mTOR(Ser2448) phosphorylation in rat hearts. Alcohol Clin Exp Res 32: 43-51, 2008.

20. Sanchez Canedo C, Demeulder B, Ginion A, et al: Activation of the cardiac mTOR/p70(S6K) pathway by leucine requires PDK1 and correlates with PRAS40 phosphorylation. Am J Physiol Endocrinol Metab 298: E761-E769, 2010. 